\title{
The Effect of SARS/COVID 19 on the Sense of Smell and Taste
}

\author{
Mini Reviet
Volume 2 Issue $2-202$
Author Details
Elnaz Shafigh ${ }^{1,}$ Mohsen Aminsobhani ${ }^{2}$, Navid Shafigh ${ }^{3 *}$
${ }^{1}$ Operative Department, Dentistry Faculty, AJA University, Tehran, Iran
${ }^{2}$ Endodontic Department, Dental School, AJA University of Medical Sciences, Tehran, Iran
${ }^{3}$ Department of Anesthesiology and Critical Care Medicine, Shahid Beheshti University of Medical Sciences, Iran
*Corresponding author
Navid Shafigh, Department of Anesthesiology and Critical Care Medicine, School of Medicine, Shahid Beheshti University of
Medical Sciences, Tehran, Iran \\ Article History \\ Received: October 23, 2021 Accepted: October 30, 2021 Published: November 22, 2021
}

\begin{abstract}
Today, changes in smell and taste are early signs of COVID-19 symptoms. Our main purpose in reviewing this article is to compare these reports and show that air olfactory dysfunction can be one of the early symptoms of COVID-19. Various causes have been reported for olfactory disorders, including inflammation of the cleft and obstruction of the nose and mouth, and damage to the epithelium of the cells that support the eye socket. The neurotropic potential of the coronavirus, which can attack and damage the olfactory bulb. In this study, we concluded that COVID requires the expression of two genes, ACE2 and TMPRESS 2, serum proteinase, and these two genes are expressed in the olfactory cells of the olfactory epithelium but are not present in the nervous system, which is why the sense of smell and taste improve after viral infection. Because the nervous system is not damaged.
\end{abstract}

Background: Today smell and taste changes are early indicators of COVID-19 symptoms. and have political decision effectiveness. An accurate matrix of disease spread is critical for the reopening strategies. Deniss person and et al reported that change in smell and taste is one of the earliest symptoms of COVID-19 that can cause self-report of disease [1]. In the other researches, the loss of smell and taste was reported as a prominent symptom of COVID-19 too [2-5].

In some countries such as Iran [6], Spain [7] France [8] Italy [9] Germany [10], and the UK [2] reported that change in smell was very dominant, and can more specific than fever and dry cough [11]. Our primary aim of this article review is to compare these reports and show that weather olfactory dysfunction could be used as an early symptom of COVID-19 and if was, why? The Mennis et al. [2] literature says that the patients who reported the loss of smell and taste were higher in those with positive test results than negative tests [2]. These data in mennis test collected by a special app that all of the susceptible patients used. Lack of smell test known as anosmia. The mechanism of action for the anosmia has been postulated [11,12]. Other studies indicated that several patients with diagnosed COVID-19 but not all of them reported anosmia [3,13] Menni reported that lack of smell caused $10-15 \%$ of patients in COVID-19 [2,14]. In the Hopkins review < in the population of the patients who are reported COVID-19 prevalence of anosmia was 62\%, and in the patients with anosmia the positive test of COVID-19 was $61 \%$ [13]. Bagheri et al. [6] in their research of 10069 patients of COVID-19 reported that $60 \%$ of patients have anosmia at their start of the disease. Gilani s et al. [15] in their research reported that patients have anosmia a few days after fever and malaise, they say that mechanism of injury for anosmia in COVID-19 is similar to other peripheral neurologic disturbances for other coronaviruses. Moen et al. [16] evaluated that 60 patients with COVID-19 completed the Persian version of smell and taste of Pennsylvania (upset) and analyzed that $98 \%$ of patients have changes olfactory dysfunction in various degrees and $25 \%$ have complete anosmia.

\section{Methods}

A systematic literature search was performed in the Medline, Pubmed, Web of Science, Embase, and Cochrane library search database using the following search expressions:

- (Olfaction disorders (MESH] AND (COVID 19 OR SARS - CoV-2 OR COVID -19 .
Another search was performed to increase the search sensitivity of the Medline database

- COVID - 19 diagnosis.

- COVID - 19 anosmia olfaction disorders.

- COVID - 19 Symptoms of clinical manifestations. 


\section{- COVID - 19 reviews.}

- Olfactory anosmia after virus.

The following terms were searched by Google: anosmia, COVID-19, and evidence-based medication.

\section{Discussion}

distinct populations have been assessed in the literature. the first group has COVID-19 positive test and prevalence of anosmia were analyzed and the second group those that have anosmia and then the prevalence of COVID-19 positive test were analyzed. In this review we ask 2 important questions:

- Why is the COVID-19 virus caused anosmia?

- Can we say that anosmia is the coronavirus's early symptoms?

In terms of the etiology of anosmia several ideas were reported:

a. Cleft inflammation and obstruction of the nose and orofacial and injury to sustentacular supporting cells epithelium.

b. Neurotropic potential of coronavirus that this virus can invade and damage the olfactory bulb. saussez et al. [17] in their research show that sinus mucosa of 16 patients in their study was clean and no thickening mucosa was observed in CT images olfactory mucosa were clear in 7 patient's and 6patient's partial opacified seen and in 3 other patients sinus space were completely opacified. Chen et al. [18] showed that ACE2 receptors (that were SARS COVID 19 receptors) immunohistochemical expression was 200 to 700 times greater in the olfactory neuroepithelium than nasal or tracheal epithelium and this can show that why COVID 19 invade this epithelium earlier and much worse. In the saussez study by using colonial antibody they find modified and expanding stained epithelium cells particularly in the nasal and oral mucosa, salivary gland, and vocal cord, and weaker in tonsil and pharynx and laryngeal supra glutes epithelium [17]. This issue shows that why these patients have anosmia and lack of taste sensation. Despite the potential mechanism of epithelium injury, nasal cytological studies in 12 patients with COVID reported that all of them have smell impairment and lacked typical findings of typical cellular injury [19].

Therefore the first idea of epithelium injury cannot likely account for anosmia. Suzuki et al. [20] in 2007 demonstrated that some patients infected with coronavirus family didn't recover their olfaction but they have a normal acoustic rhinotomy which suggests that nasal inflammation and obstruction were not early etiology of anosmia. Before this shown that coronavirus is one of the latest viruses that can enter the brain through the olfactory bulb and can rapid trans neural spread [21].

The authors of this research demonstrated that regions of the cortex (piriform and infralimbic) basal ganglia and midbrain (dorsal raphe) were infected after the virus spreading [21]. Saussez et al. [17] analyses that in mild cases of COVID 19 patients have stronger local immunity such as anosmia and in a severe form that lead to hospitalization patients have less report of ENT symptoms and anosmia. Bunyavanich collected epithelial cells of the nasal region in 305 patients that were 4 to 60 years old $.48 / 9 \%$ of these patients were male. these cells were collected by using a cytologic brush and stored in -80 RNA stabilization fluid [22]. They showed that linear regeneration of Gene ACE2 in younger patients was significantly lower than in adults in children under 10 years old Gene ACE2 was an indifferent expression and it seems that because of this SARS COVID 19 doesn't dangerous in children [22]. This study showed that the expression of ACE2 was independent of sex. It seems that in severe forms of COVID-19 infected olfactory receptor neurons may prevent anterograde propagation of virus to the olfactory bulb and central nervous system [17]. Haehner et al. [10] reported that the frequency of olfactory disorders in coronavirus is so far lower than other viruses.

But the smell and taste loss have been observed in 8-50\% of COVID patients and sometimes as the only apparent symptoms in the younger patients. Heanher et al. [10] in their study evaluated that between 500 patients with cold symptoms 69 patients have olfactory dysfunction and smell and taste loss. means $13 / 8 \%$ [10] but out of 34 patients with COVID-19 positive test 22 of them (64/7\%) have a sudden taste and small loss. Only one patient reported isolated taste loss. In this study patients with the smell, the loss was rarely accompanied by a blocked nose, and thus the effect of olfactory may be independent of nasal congestion and was a neural problem [10]. In the final article, we reviewed Bhattacharjee and et al in their study reported that $82 \%$ of asymptomatic COVID 19 patients carried olfactory defects but in the symptomatic patients only $15 \%$ have olfactory disorders [23]. They reported that virus-induced impairments of shot down of olfactory sensor neurons (ONS)may vary depending on the severity of virus loud and infection Bhattacharjee et al. [23] in their study present a novel method to assess the olfactory fitness of asymptomatic COVID 19 patients. they used a custom-built olfactory action meter and submitted that covid 19 infected not neuronal supporting cells in the olfactory epithelium and therefore it's very likely that olfactory dysfunction varies by viral loud. SARS/COVID requires the coexpression of ACE2 and TMPRESS 2 (transmembrane protease serine 2) and these 2 Genes are expressed in sustentacular cells of olfactory epithelium but not in the neural system [23]. This is the reason that after viral infection the sense of smell and taste becomes better because the neural system doesn't damage.

\section{References}

1. Pierron D, Loth VP, Mantel M, Moranges M, Bignon E, et al. (2020) Smell and taste changes are early indicators of the COVID-19 pandemic and political decision effectiveness. Nature communications 11(1): 5152.

2. Menni C, Valdes AM, Freidin MB, Sudre CH, Nguyen LH, et al. (2020) Real-time tracking of self-reported symptoms to predict potential COVID-19. Nature medicine 26(7): 1037-1040.

3. Eliezer M, Hautefort C, Hamel AL, Verillaud B, Herman P, et al. (2020) Sudden and complete olfactory loss of function as a possible symptom of COVID-19. JAMA otolaryngology head \& neck surgery 146(7): 674675 .

4. Gautier JF, Ravussin Y (2020) A new symptom of COVID-19: loss of taste and smell. Obesity (Silver Spring) 28(5): 848.

5. Pellegrino R, Cooper KW, Pizio AD, Joseph PV, Bhutani S, et al. (2020) Coronaviruses and the chemical senses: past, present, and future. Chemical senses 45(6): 415-422.

6. Bagheri SH, Asghari A, Farhadi M, Shamshiri AR, Kabir A, et al. (2020) Coincidence of COVID-19 epidemic and olfactory dysfunction outbreak in Iran. Medical journal of the Islamic Republic of Iran 34: 62.

7. Beltrán $\bigotimes$ Corbellini Á, Chico-García JL, Martínez-Poles J, RodríguezJorge F, Natera-Villalba E, et al. (2020) Acute冈onset smell and taste disorders in the context of COVID囚19: a pilot multicentre polymerase chain reaction-based case control study. European journal of neurology 27(9): 1738-1741.

8. Bénézit F, Turnier PL, Declerck D, Paillé C, Revest M, et al. (2020) Utility of hyposmia and hypogeusia for the diagnosis of COVID-19. The Lancet Infectious Diseases 20(9): 1014-1015.

9. Giacomelli A, Pezzati L, Conti F, Bernacchia D, Siano M, et al. (2020) Self-reported olfactory and taste disorders in patients with severe acute respiratory coronavirus 2 infection: a cross-sectional study. Clinical Infectious Diseases 71(15): 889-890. 
10. Haehner A, Draf J, Dräger S, With KD, Hummel T (2020) Predictive value of sudden olfactory loss in the diagnosis of COVID-19. Orl 82(4): 175-180.

11. Brann DH, Tsukahara T, Weinreb C, Lipovsek M, Van den Berge K, et al. (2020) Non-neuronal expression of SARS-CoV-2 entry genes in the olfactory system suggests mechanisms underlying COVID-19associated anosmia. Science advances 6(31): eabc5801.

12. Sungnak W, Huang N, Bécavin C, Berg M, Queen R, et al. (2020) SARSCoV-2 entry factors are highly expressed in nasal epithelial cells together with innate immune genes. Nature medicine 26(5): 681-687.

13. Rocke J, Hopkins C, Philpott C, Kumar N (2020) Is loss of sense of smell a diagnostic marker in COVID囚19: a systematic review and meta analysis. Clinical Otolaryngology 45(6): 914-922.

14. Richardson S, Hirsch JS, Narasimhan M, Crawford JM, McGinn T, et al. (2020) Presenting characteristics, comorbidities, and outcomes among 5700 patients hospitalized with COVID-19 in the New York City area. Jama 323(20): 2052-2059.

15. Gilani S, Roditi R, Naraghi M (2020) COVID-19 and anosmia in Tehran, Iran. Medical hypotheses 141: 109757.

16. Moein ST, Hashemia SMR, Mansourafshar B, Khorram-Tousi A, Tabarsi P, et al. (2020) Smell dysfunction: a biomarker for COVID囚19. International forum of allergy \& rhinology 10(8): 944-950.

17. Saussez S, Lechien JR, Hopkins C (2020) Anosmia: an evolution of our understanding of its importance in COVID-19 and what questions remain to be answered. European Archives of Oto-Rhino-Laryngology $1-5$.

18. Chen M, Shen W, Rowan NR, Kulaga H, Hillel A, et al. (2020) Elevated $\mathrm{ACE}_{2}$ expression in the olfactory neuroepithelium: implications for anosmia and upper respiratory SARS-CoV-2 entry and replication. European Respiratory Journal 58(5).

19. Gelardi M, Notargiacomo M, Trecca EMC, Cassano M, Ciprandi G (2020) COVID-19 and nasal cytobrush cytology. Acta Cytologica 64: 397-398.

20. Suzuki M, Saito K, Min WP, Vladau C, Toida K, et al. (2007) Identification of viruses in patients with postviral olfactory dysfunction. The Laryngoscope 117(2): 272-277.

21. Netland J, Meyerholz DK, Moore S, Cassell M, Perlman S (2008) Severe acute respiratory syndrome coronavirus infection causes neuronal death in the absence of encephalitis in mice transgenic for human $\mathrm{ACE}_{2}$. Journal of virology 82(15): 7264-7275.

22. Bunyavanich S, Do A, Vicencio A (2020) Nasal gene expression of angiotensin-converting enzyme 2 in children and adults. Jama 323(23): 2427-2429.

23. Bhattacharjee AS, Joshi SV, Naik S, Sangle S, Abraham NM (2020) Quantitative assessment of olfactory dysfunction accurately detects asymptomatic COVID-19 carriers. EClinicalMedicine 28: 100575 . 